\title{
Intervista a Sandro Bandini
}

\author{
Sandro Bandini, MD \\ Nefrologia dei Trapianti e Dialisi \\ Azienda Ospedaliera-Universitaria Careggi, Firenze \\ sandro.bandini1@virgilio.it
}

Nel centro ove presti attività qual è la percentuale di pazienti monitorati per CKD-MBD stadio 3-5 e quali marker biologici usi a tal fine?

Nel nostro ambulatorio nefrologico tutti i pazienti con malattia renale cronica, stadio 3-5 vengono preliminarmente sottoposti a valutazione iniziale della calcemia, fosforemia, PTH intatto, fosfatasi alcalina e 25(OH) D. Nelle visite successive con periodicità media tri-quadrimestrale per i pazienti con CKD grado 3-4 vengono sempre valutati calcemia e fosforemia. Il PTH e la fosfatasi alcalina vengono determinati mediamente tre volte all'anno. Ovviamente in base alla progressione della malattia renale, ai valori iniziali del PTH e al trattamento farmacologico effettuato (dose di vitamina D somministrata), la frequenza delle determinazioni è maggiore fino a un controllo adeguato dei parametri considerati. In presenza di malattia renale cronica, stadio 5 noi effettuiamo un ambulatorio dedicato alla "Pre Uremia" che prevede controlli ematochimici più ravvicinati.

Nel centro ove presti attività pratichi il dosaggio della $25(\mathrm{OH}) \mathrm{D}$ e/o della $1,25(\mathrm{OH})_{2} \mathrm{D} 3$ ?

Attualmente effettuiamo, come suggerito dalle linee guida KDIGO, il dosaggio della $25(\mathrm{OH}) \mathrm{D}$ a tutti i pazienti che afferiscono al nostro centro: sia a pazienti con CKD 3-5 sia a pazienti in trattamento dialitico. Di norma analizziamo i livelli di tale vitamina due volte all'anno (inizio e fine anno). Nei soggetti trattati la frequenza può incrementare. Non riteniamo utile praticare il dosaggio dell'1,25(OH) ${ }_{2} \mathrm{D} 3$, per l'alto costo e soprattutto perché non modificherebbe il nostro approccio terapeutico alla malattia ossea.

Applichi la correzione di 25(OH)D in caso di carenza? Se si, in quale tipo di pazienti e in quale percentuale?

Per livelli di 25(OH)D $<30 \mathrm{ng} / \mathrm{mL}$ (insufficienza) o a maggior ragione in presenza di deficit somministriamo, secondo quanto riferito in letteratura, Colecalciferolo (vitamina D3) (DIBASE $(10000 \mathrm{u} / \mathrm{mL}) 3-4-5 \mathrm{gtt} / \mathrm{die}$ 750-1000 U vitamina D3). La somministrazione di Calcifediolo (Didrogyl 2-4gtt/die) può essere altrettanto usata. È utile controllare dopo 2-3 mesi di terapia i livelli di $25(\mathrm{OH}) \mathrm{D}$. Il tentativo è la correzione almeno parziale del deficit nutrizionale che è presente in larga percentuale nei pazienti con IRC già allo stadio 3 e che incrementa con il progredire della $\mathrm{CKD}$. Si è notato inoltre, che la correzione del deficit migliora la BMD e inoltre può almeno inizialmente correggere il deficit di calcitriolo. L'utilizzo della $25(\mathrm{OH}) \mathrm{D}$ non preclude anzi integra, come suggerito dalla linee guida KDIGO, la somministrazione di calcitriolo, favorendone l'azione inibitoria sulla secrezione di ormone paratiroideo.

Nel centro ove presti attività in quale percentuale o in quali casi clinici particolari pratichi la biopsia ossea?

L'indagine istologica dell'osso mediante esame istomorfometrico, rappresenta, come è noto, il "Gold Standard" per una sicura diagnosi di patologia ossea. Le linee guida KDIGO suggeriscono la biopsia ossea particolarmente in presenza di: fratture, ipercalcemia e ipofosfatemia di non chiara origine e nei casi che necessitino di terapia con bifosfonati allo scopo di escludere la presenza di una Adinamic Bone Disease che controindica tale trattamento.

Quest'ultima raccomandazione è utile perché a differenza di qualche anno fa in cui l'uso di questi farmaci era limitato al trattamento dell'ipercalcemia secondaria a IPT autonomo, oggi ne viene consigliata la somministrazione anche nei pazienti con CKD di grado elevato (4-5). Nel nostro centro non effettuiamo la biopsia ossea da vari anni in cui inviavamo il reperto istologico da esaminare ai pochi centri in Italia in grado di effettuare una valutazione non solo qualitativa ma anche quantitativa mediante l'istomorfometria ossea. Attualmente credo che per l'invasività della biopsia ossea che necessita di più campioni ossei per singolo esame e che 
comunque a distanza di tempo va ripetuta, per gli alti costi dovuti all'utilizzo di attrezzature sofisticate, sia di fatto impossibile eseguire questa metodica diagnostica nella pratica clinica.

Nel centro ove presti attività, in quale percentuale o in quali stadi di CKD usi indagini strumentali per la ricerca di calcificazioni vascolari? Quali indagini utilizzi?

È noto che le calcificazioni cardiovascolari sono associate ad aumentata morbilità e mortalità nel paziente uremico. Pertanto vanno sicuramente indagate precocemente e sarebbe auspicabile, ma estremamente difficile, agire preventivamente.

I nostri pazienti con stadio di malattia renale cronica 3-5 e in trattamento emodialitico, presentano una prevalenza di calcificazioni vascolari elevata con arteriopatie periferiche frequenti e disabilitanti. Tale incidenza è aumentata negli ultimi 4-5 anni soprattutto per l'incremento dell'età anagrafica della popolazione sottoposta a dialisi.

Non eseguiamo indagini sulle calcificazioni vascolari, sistematicamente su tutti i pazienti, con periodicità omogenea. In base all'età anagrafica e dialitica del paziente, la presenza di comorbidità, parametri bioumorali, eventi acuti clinici, eseguiamo Rx di alcuni segmenti ossei, ecocardiogramma, ecocolor Doppler dei vasi del collo e arti inferiori. Da circa un anno effettuiamo, in accordo con i suggerimenti delle KDIGO, Rx laterale dell'addome per la valutazione delle calcificazioni dei grossi vasi.

Nel centro ove presti attività, qual è la percentuale di pazienti in trattamento sostitutivo che resta nel range di normalità secondo le linee guida SIN per Ca, P, e PTH?

Abbiamo effettuato in tal senso nel nostro centro, nel 2009, una indagine su 130 pazienti in dialisi extracorporea. Tenendo presente le linee guida DOQI del 2003 che poco differiscono dalle linee guida della SIN abbiamo evinto i seguenti dati:

- il 30\% dei nostri pazienti presentava livelli di $\mathrm{P}>$ di 5,5 $\mathrm{mg} / \mathrm{dL}$; il $51 \%$ dei soggetti rientrava nel range di normalità delle KDOQI ( $\mathrm{P}=3,5-5,5 \mathrm{mg} / \mathrm{dL})$;

- il 55\% dei pazienti presentava valori di PTH nel range di normalità $(\mathrm{PTH}=150-300 \mathrm{pg} / \mathrm{mL})$; il $18 \%$ aveva livelli di paratormone $>$ di $300 \mathrm{pg} / \mathrm{mL}$, il $27 \%$ valori di $\mathrm{PTH}<$ $300 \mathrm{pg} / \mathrm{mL}$;

- il 10\% presentava livelli di calcemia $>9,6 \mathrm{mg} / \mathrm{dL}$. Ipocalcemia $(\mathrm{Ca}<8,2 \mathrm{mg} / \mathrm{dL})$ si evidenziava nel $7 \%$ dei nostri pazienti.
Nel centro ove presti attività, qual è la percentuale dei vari chelanti usati per ricercare il controllo del Ca-P nei pazienti in trattamento sostitutivo?

Nel nostro centro attualmente il 70\% circa dei pazienti utilizza chelanti dei fosfati. Calciocarbonato in monoterapia o associato a Sevelamer viene prescritto al 35\% dei nostri pazienti emodializzati. Sevelamer in monoterapia viene assunto dal 40\% circa dei soggetti. Il 18\% dei pazienti utilizza carbonato di lantanio. Una minima percentuale di uremici assume sali di alluminio per brevi periodi di tempo e nei casi di resistenza agli altri chelanti.

Nel centro ove presti attività qual è la percentuale di pazienti in CKD 3-5 sottoposta a dieta ipoproteica per il controllo della iperfosforemia?

La dieta ipoproteica (con o senza prodotti aproteici) viene prescritta a una percentuale elevata nei nostri pazienti con CKD 3-5 e viene controllata da un servizio di dietologia accurato. I pazienti se necessario compilano un diario dietetico settimanale ed effettuano visite ambulatoriali con periodicità variabile a secondo delle necessità. Sono richiesti controlli ematochimici e urinari atti a comprendere la compliance alla dieta (urea siero, urine fosforemia, fosfaturia ecc.). Nelle fasi più avanzate della malattia renale cronica, a una percentuale limitata di pazienti (particolarmente motivati) viene prescritta dieta vegetariana associata a chetoanaloghi.

Se il paziente è compliante al trattamento, si riescono a ottenere ottimi risultati nel controllo dell'acidosi metabolica, dei livelli di fosforemia, e non ultimo dei livelli di paratormone.

Nel centro ove presti attività quale percentuale di pazienti in trattamento sostitutivo vitamina $D$ o analoghi, calciomimetici o entrambi?

Le percentuali si riferiscono ai pazienti totali in trattamento dialitico.

La vitamina $\mathrm{D}$ è prescritta nel $58 \%$ circa dei pazienti in trattamento emodialitico.

Nel nostro centro il 18\% dei pazienti assume Cinacalcet. Il Cinacalcet è associato ad analoghi della vitamina $D$ nel $50 \%$ dei casi. Il Cinacalcet in monoterapia è utilizzato per lo più nei pazienti di difficile gestione con livelli di PTH medio-alti non ben controllabili e con valori di calcemia e fosforemia spesso al di sopra dei limiti della norma. Il paracalcitolo viene usato nel $28 \%$ dei pazienti. Il 30\% dei pazienti assume calcitriolo orale. 
Dopo oltre un trentennio di osservazioni, esperienze, studi, ricerche, la fisiopatologia dell'iperparatiroidismo secondario non è ancora del tutto chiarita. Calcio, fosforo, recettori della vitamina $D$, recettori sensibili al calcio, ormoni fosfatotropi, sono tutti "in carriera" insieme al primo attore il PTH (almeno nella cronologia storica): si $\grave{e}$ in grado di prevedere un ordine e un chiarimento delle cose o dobbiamo ancora e solo predire?

La fisiopatologia dell'iperparatiroidismo secondario del paziente uremico è sicuramente un "puzzle" ancora tutto da definire.

Pur rimanendo i bassi livelli di calcio, il deficit di calcitriolo e la ritenzione dei fosfati fattori patogenetici essenziali dell'aumentata sintesi e iperplasia della ghiandola paratiroidea, studi più recenti effettuati su modelli animali in vitro e in vivo e sull'uomo, hanno condotto a una migliore conoscenza dei meccanismi "effettori" della loro azione sulla regolazione della funzione paratiroidea, vedi il ruolo meglio definito dei recettori per il calcio (CaSR), e per la vitamina D (VDR).

Recentemente questo complesso scenario si è arricchito di un nuovo attore: il complesso FGF23/ KLOTHO. Entrambi sono stati coinvolti nella fisiopatologia delle alterazioni del metabolismo minerale del paziente uremico. L'FGF23 (fattore di crescita fibroblastico), è una proteina prodotta dal tessuto osseo (osteociti/osteoblasti) appartenente al gruppo delle fosfatonine, che regola l'omeostasi fosfatica, inducendo fosfaturia per inibizione del cotrasporto sodio-fosfato (NaPi2a) a livello del tubulo renale prossimale e ridotto assorbimento intestinale di fosforo, mediante inibizione dell'1alpha idrossilasi renale e riduzione dei livelli di calcitriolo (1). La regolazione sistemica della sintesi di FGF23 è mediata dalla $1,25(\mathrm{OH})_{2} \mathrm{D} 3$ che attiva il promotore genico di FGF-23, dai livelli sierici della fosforemia e probabilmente dai livelli di calcemia e PTH (2).

L'attività endocrina dell'FGF-23 si esplica mediante l'interazione con uno specifico recettore di membrana ad attività tirosinchinasica, l'FGF-R (recettore ubiquitario) in presenza del suo cofattore la proteina KLOTHO (espressa prevalentemente su rene e paratiroide), la cui presenza è indispensabile per potenziare l'affinità dell'FGF23 col proprio recettore e consentirne l'attività biologica. FGF23 e KLOTHO oltre che interagire come complesso, possono esercitare effetti diretti indipendenti: FGF23 esercita una funzione locale (paracrina) a livello osseo di inibizione della maturazione degli osteoblasti mentre KLOTHO stimola il riassorbimento renale del calcio agendo sui cosiddetti "canali del calcio" (TRPV5) e sembra regolare secondo le necessità la produzione di paratormone (studi effettuati su modelli animali ipocal- cemici) (3). Questa interrelazione stretta tra i due fattori ha indotto alcuni autori a introdurre il concetto di "Asse Funzionale OSSO-RENE” (2).

E stato recentemente documentato che il sistema "FGF23/KLOTHO" esercita un'azione di inibizione diretta della secrezione di PTH. Due studi effettuati in vivo e in vitro su modelli animali e colture di cellule paratiroidee, dimostrano che l'FGF23 agisce sulla ghiandola paratiroidea inibendo la produzione di PTH mediante attivazione tirosinchinasica (ERK-MAPkinase pathway) $(4,5)$. In particolare, incubando cellule paratiroidee bovine con dosi crescenti di FGF23 ricombinante, è stato evidenziato che FGF23 inibisce l'RNAmessaggero per il PTH e la secrezione del paratormone, ma un po' sorprendentemente, stimola localmente, invece di sopprimere, come si verifica a livello renale, la produzione di 1alpha idrossilasi. Alla luce di questi dati preliminari così intriganti, Silver (2) delinea un secondo Asse Funzionale "Osso-Paratiroide" (Bone parathyroid endocrinological feed-back loop) la cui importanza nel complesso scenario del metabolismo minerale deve ancora essere pienamente valutata.

Secondo dati recenti della letteratura, il ruolo di FGF23/ KLOTHO nella fisiopatologia dell'IPTS sarebbe assai rilevante: vari autori, infatti, hanno evidenziato un coinvolgimento di tale sistema nelle fasi molto precoci della CKD.

Gutierrez (6) in uno studio su pazienti con CKD normofosforemici, ha osservato un aumento costante dei livelli di FGF23 con il progredire della insufficienza renale a partire da livelli di VFG di $60 \mathrm{~mL} / \mathrm{min}$. È stato documentato che l'FGF23 incrementava già con valori di VFG di $90 \mathrm{~mL} / \mathrm{min} / 1,73 \mathrm{~m}^{2}$, in presenza di normali livelli di calcitriolo e di PTH (7). Evenepoel (8), in uno studio cross sectional su pazienti normofosforemici, con CKD (1-3) ha evidenziato che il $61 \%$ di essi presentava valori di FGF23 superiori alla norma e solo il $10 \%$ valori di PTH elevati e che l'escrezione dei fosfati correlava con livelli elevati di FGF23 e PTH. Oliveira (9) ha dimostrato, anche se in uno studio di breve durata, che l'utilizzo dei chelanti dei fosfati (sevelamer, calcioacetato) nella "early CKD", è in grado di ridurre l'escrezione urinaria del P, i livelli di PTH, ma che solo sevelamer riduceva la produzione di FGF23. Infine un'analisi istochimica condotta su tessuto osseo di pazienti con CKD di grado 2, ha rilevato a livello osteocitario una aumentata espressione di FGF23 rispetto ai controlli (10).

Queste recenti acquisizioni, seppure tutte da confermare, hanno messo in discussione la teoria "classica" secondo cui l'aumentata secrezione di PTH sarebbe la diretta conseguenza del deficit precoce di $1,25(\mathrm{OH})_{2} \mathrm{D} 3 \mathrm{e}$ hanno rivalutato il ruolo della ritenzione dei fosfati come 
fattore determinante o per meglio dire primum movens per lo sviluppo di un IPTS ; si parla al riguardo infatti di "Teoria Fosforocentrica".

Secondo questa nuova interpretazione patogenetica, la diminuita escrezione di fosfati conseguente alla ridotta massa nefronica, determinerebbe un'aumentata produzione osteocitaria di FGF23 che per mantenere l'omeostasi del fosforo ne aumenterebbe l'escrezione urinaria e ne ridurrebbe l'assorbimento intestinale, inibendo la sintesi di calcitriolo (il cui deficit sarebbe il prezzo da pagare per mantenere livelli normali di fosforemia). Lipocalcemia calcitriolo dipendente stimolerebbe successivamente secondo la teoria classica la secrezione di ormone paratiroideo.

Una questione però sembrerebbe irrisolta: se l'FGF23 controlla nei soggetti normali, inibendola la secrezione di PTH, perché i pazienti con CKD sviluppano IPTS nonostante gli alti livelli di FGF23? La spiegazione di questa resistenza sembra risiedere in una ridotta espressione del complesso recettoriale KLOTHO-FGFR1 a livello del tessuto paratiroideo iperplastico evidente, soprattutto, in presenza di un quadro istologico di iperplasia nodulare, così come è stato dimostrato per i recettori del calcio (CaSR) e della vitamina D (VDR). Vari studi effettuati sull'uomo e sui ratti confermano questa ipotesi patogenetica: Komaba (11) mediante tecnica istochimica ha rilevato una down-regulation di KLOTHO e FGFR1 nel tessuto ghiandolare iperplastico di 25 pazienti con CKD 5. Kumata (12) sull'uomo, e Canalejo, Galitzer $(13,14)$ sui ratti, hanno confermato i dati di Komaba. In particolare Galitzer, utilizzando tessuto paratiroideo di ratto uremico e somministrando FGF23 ricombinante, ha rilevato che l'azione di regolazione del PTH da parte della fosfatonina, si mantiene solo negli stadi iniziali della insufficienza renale, ma non negli stadi avanzati (CKD 5), per la down regulation del complesso KLOTHO/FGFR1.

$\mathrm{Ci}$ sono ancora ovviamente tante domande da porci relativamente al ruolo dell'FGF23 nella malattia renale cronica e tutte le ipotesi avanzate devono essere per lo più ancora validate.

- Non sono ancora chiarite le modalità con cui il fosforo regola la produzione di FGF23: non è stato identificato un recettore cellulare per il fosforo.

- Non sappiamo qual è il normale range di normalità dell'FGF23 nei vari stadi di CKD, così come avviene per il PTH.

- Elevati livelli di FGF23 nella CKD correlano con una varietà di condizioni cliniche come l'ipertrofia ventricolare sinistra, le calcificazioni vascolari e addirittura con un aumentato rischio di mortalità: qual è allora il reale ruolo
dell'FGF23, marker o fattore patogenetico tossico diretto sui tessuti?

- Quando dobbiamo trattare e a che livello di fosforemia i nostri pazienti per controllare la secrezione osteocitaria di FGF23? Se è vera la teoria "Fosforocentrica" bisogna intervenire precocemente e trattare i pazienti normofosforemici, con CKD agli stadi iniziali, mediante una dieta controllata in fosforo o con l'impiego di chelanti dei fosfati, come è suggerito da alcuni lavori preliminari $(9,15)$.

A di là dei dubbi legittimi sollevati da queste domande, l'individuazione di questo nuovo sistema ormonale FGF23/KLOTHO nella complessa patogenesi dell'iperparatiroidismo secondario, ha aperto nuove prospettive terapeutiche delle alterazioni del metabolismo minerale del paziente con malattia renale cronica.

Perché supplementare con calcifediolo e calcitriolo $i$ pazienti con CKD 3-5, con carenza di 25(OH)D?

Insufficienza e deficit di vitamina $\mathrm{D}$ sono presenti in larga percentuale nella popolazione normale e nei pazienti con CKD 3-5. Studi recenti hanno dimostrato che con il progredire della IRC si riducevano parallelamente i livelli di $25(\mathrm{OH}) \mathrm{D}$ e addirittura tale deficit interessava la quasi totalità dei pazienti in dialisi (16). Le cause di questa carenza sono riconducibili prevalentemente a un deficit nutrizionale, a ridotta sintesi cutanea per non adeguata esposizione solare, a perdita renale in presenza di proteinuria nefrosica. Le KDIGO suggeriscono di misurare i livelli di $25(\mathrm{OH}) \mathrm{D}$ e di tentare di correggere la «vitamin D deficiency $(<15-10 \mathrm{ng} / \mathrm{mL})$ and insufficiency (15-30 ng/ $\mathrm{mL}$ ) in accordance to treatment strategies reccomended for the general population» (17). Ci sono però altre considerazioni che devono indurre il nefrologo all'uso della vitamina D a scopo nutrizionale nella IRC. La $25(\mathrm{OH})$ $\mathrm{D}$ ha un effetto diretto sulla mineralizzazione scheletrica indipendentemente dai livelli di 1,25(OH)2D3 (18). I bassi livelli di calcifediolo sono stati associati a rischio di aumentata morbilità cardiovascolare e mortalità nel paziente con malattia renale cronica, senza considerare gli effetti specifici sul sistema immunitario (19).

Infine conseguenza del deficit di calcifediolo è l'accentuazione del deficit anche della 1,25(OH)2D3 per ridotta conversione enzimatica da parte della 1alfa-idrossilasi della $25(\mathrm{OH}) \mathrm{D}$ : quindi la somministrazione di vitamina D contribuirebbe a ridurre i livelli di PTH. In alcuni studi, infatti, la normalizzazione dei livelli di $25(\mathrm{OH}) \mathrm{D}$ a pazienti con CKD 3-4 ha determinato un miglior controllo dei livelli di PTH(20). Ovviamente, per un adeguato controllo dell'IPTS è indispensabile la somministrazione di calcitriolo o dei suoi analoghi (paracalcitolo) (21). Levin 
ha evidenziato, infatti, una prevalenza elevata di deficit di 1,25(OH)2D3 dallo stadio 2 allo stadio 4 (dal $20 \%$ al $75 \%$ ) anche in presenza di normali livelli di PTH negli stadi precoci di malattia (22). A questo proposito nei pazienti con CKD 3-5, le linee guida KDIGO suggeriscono, in presenza di livelli di PTH sopra la norma e "progressively rising", la somministrazione di calcitriolo o analoghi della vitamina D. Il paracalcitolo, attivatore selettivo dei VDR, in particolare, per i suoi effetti pleiotropici e per la minor incidenza di episodi di ipercalcemia e iperfosforemia testimoniati da numerosi contributi della letteratura, sembra farsi preferire al calcitriolo $(23,24)$.

\section{1-84;1-34;36-84;7-84 quale paratormone per quale recet- tore e per quale paziente?}

Come è noto il paratormone 1-84 è la forma biologicamente attiva dell'ormone prodotta dalle ghiandole paratiroidi e secreta in circolo. Il PTH esercita la sua azione mediante l'interazione dei primi 34 aminoacidi della regione $\mathrm{N}$ terminale (biologicamente attiva) con il recettore tipo 1 PTH/PTHrP (PTHR1).

Oltre alla molecola intera PTH 1-84, in circolo sono presenti frammenti della molecola paratiroidea carbossiloterminale $(\mathrm{C})$ o aminoterminale $(\mathrm{N}$ terminal) che derivano o dalla proteolisi intraghiandolare del paratormone o dalla sua degradazione periferica (epatica/renale): la proteolisi verificandosi in posizioni diverse della molecola dà origine a una grande varietà di frammenti. I frammenti $\mathrm{C}$ terminali sono percentualmente in quantità elevata (70-95\%), al contrario dei frammenti $\mathrm{N}$ terminali troncati. Sono stati identificati recentemente 4 frammenti N-troncati di dimensioni simili al frammento più rappresentativo 7-84 che interagirebbero a livello osteocitario con recettori specifici (C-PTH receptor) riducendo il riassorbimento osseo. Il rilievo di questi frammenti che modulano, inibendola, l'azione dell'ormone paratiroideo, potrebbe, secondo alcuni autori, aggiungere valore diagnostico al semplice dosaggio della molecola intera. Negli anni Ottanta i metodi RIA di I generazione rilevavano i frammenti C-terminali e medio molecolari sovrastimando, nei pazienti con CKD, i livelli di PTH, per la prevalente clearance renale di tali frammenti; anche il dosaggio del solo frammento N-terminale (1-34), non si dimostrò attendibile per la sua scarsa concentrazione ematica e breve emivita. L'introduzione nei primi anni Novanta dei metodi immunoradiometrici di II generazione (Allegro della Nichols, capostipite), migliorò nettamente la sensibilità e la specificità del dosaggio del paratormone. Essi, infatti, misuravano il cosiddetto PTH Intatto, utilizzando un atc specifico per la porzione $\mathrm{N}$ terminale marcato con Iodio 12I e un atc diretto contro la porzione C-terminale 35-84 della molecola paratiroidea; negli anni successivi, ai metodi di dosaggio IRMA si sono aggiunti i metodi chemiluminometrici ICMA. I metodi di II generazione sono i più diffusi anche oggi, tant'è che nel corso degli anni vari kit di dosaggio commerciali si sono resi disponibili; ma anch'essi hanno mostrato problemi di specificità. Era stato, infatti, supposto per qualche tempo che il "PTH Intatto" rappresentasse la sola molecola intera; in realtà è stato successivamente dimostrato che con queste metodiche, venivano dosati anche i frammenti $\mathrm{C}$ terminali $\mathrm{N}$-troncati, comunemente definiti frammenti 7-84. Il rischio è quindi la sovrastima del grado di iperparatiroidismo, con possibilità di errori terapeutici da parte del nefrologo. Il problema sembrerebbe superato dall'avvento dei metodi di III generazione che hanno la caratteristica di rilevare specificamente il cosiddetto PTH “intero" (whole PTH). Essi utilizzano come tracciante un atc diretto verso la porzione 1-5 della molecola paratiroidea e quindi senza possibilità di interferenza con i frammenti cosiddetti 7-84 $\mathrm{N}$ troncati in posizione precoce, che crossreagiscono con gli assays per il PTH Intatto. I valori ottenuti con il dosaggio del paratormone intero sono, infatti, inferiori del $30-40 \%$ rispetto ai valori del PTH Intatto. Questa metodica, ha permesso di identificare un frammento N-terminale (N-PTH) di incerto significato clinico, che rappresenta il 4-8\% del PTH circolante nei soggetti normali, fino a più del $20 \%$ nei soggetti con iperparatiroidismo primitivo o secondario. Se i risultati del dosaggio sono importanti, altrettanto importante è l'affidabilità del dosaggio. Confrontando i diversi metodi di II generazione (IRMA-ICMA) per il PTH Intatto tra di loro, si è notata una valida correlazione tra $\mathrm{i}$ metodi, ma un differenza notevole tra i valori assoluti di PTH misurato; anche i metodi di III generazione, attualmente disponibili, che dovrebbero misurare il vero PTH hanno mostrato i medesimi problemi. Queste variazioni significative di valori dei livelli di paratormone misurato, derivano da una diversa specificità degli atc usati e dal fatto che non esiste ancora un metodo validato come Golden Standard per il dosaggio del PTH (25).

È stato documentato (26) come utilizzando diversi metodi di dosaggio dell'ormone paratiroideo nello stesso paziente (II-III generazione) in trattamento con paracalcitolo o calciomimetico, la decisione di modificare o sospendere la terapia può differire in modo significativo a seconda del kit usato per il dosaggio.

Quale metodo preferire? Ancora non esiste accordo in letteratura: i metodi di III generazione specifici per la molecola intera, valutano sicuramente in modo più preciso i livelli del "Vero PTH"; non sembrano migliorare però il valore predittivo del tipo di patologia ossea rispetto ai metodi classici che utilizzano il dosaggio del PTH 
Intatto. Le linee guida KDIGO a questo proposito suggeriscono di continuare a utilizzare nella pratica clinica $\mathrm{i}$ metodi classici di II generazione (17).

Pertanto, per una corretta valutazione clinica, spetta al nefrologo standardizzare i metodi di prelievo, stoccaggio dei campioni, il tipo di campione da analizzare (siero/plasmaEDTA: influenza il dosaggio) e soprattutto conoscere bene, in accordo con il laboratorio, le caratteristiche del kit usato per la determinazione del paratormone.

Calciomimetici: potenti, affidabili, indispensabili: quando, come e perché?

I calciomimetici sono farmaci che aumentano la sensibilità del CaSR presente sulla ghiandola paratiroidea al calcio, regolando la secrezione dell'ormone. Numerosi studi prospettici randomizzati effettuati in pazienti con lieve-severo IPTS hanno dimostrato che il calciomimetico associato alla terapia tradizionale con vitamina $\mathrm{D}$, incrementava la percentuale di pazienti che rispettavano le linee guida KDOQI, per quanto riguarda i livelli di PTH, calcemia e fosforemia.

Lo studio OPTIMA in particolare ha valutato oltre 500 pazienti, con valori di PTH compresi tra $300-800 \mathrm{pg} / \mathrm{mL}$, randomizzati a terapia convenzionale o a terapia combinata con Cinacalcet. Il $71 \%$ dei pazienti trattati anche con calciomimetico ha raggiunto livelli di PTH $<300 \mathrm{pg} / \mathrm{mL}$ (endpoint primario) versus solo il $22 \%$ del gruppo di controllo. Da notare che nei pazienti che assumevano Cinacalcet si assisteva a una riduzione progressiva della dose di vitamina $\mathrm{D}$ somministrata. In alcuni casi l'introduzione del Cinacalcet in terapia determinava un incremento dei pazienti che dovevano assumere chelanti a base di calcio, per l'ipocalcemia Cinacalcet-dipendente (attenzione a monitorare i livelli di calcemia nei pazienti che assumono il farmaco) (27). Un altro studio (ACHIEVE) ha valutato su 87 pazienti trattati per 33 settimane, l'efficacia di Cinacalcet associato a una dose fissa di paracalcitolo rispetto a un altro gruppo di 86 soggetti trattati con dosi variabili di vitamina $\mathrm{D}$ a secondo delle necessità. Nel $43 \%$ circa dei pazienti che assumevano calciomimetico si otteneva una riduzione dei livelli di PTH $<300 \mathrm{pg} / \mathrm{mL}$ vs il $23 \%$ nel gruppo di soggetti cui era somministrata vitamina D (28). Da notare infine che da uno studio osservazionale, europeo, recente, si evince che l'efficacia di Cinacalcet si manifesta in modo rilevante nei pazienti con IPT medio-severo che non possono assumere dosaggi adeguati di vitamina D per gli elevati livelli di calcemia e fosforemia (29). Uno studio è in corso (EVOLVE) per valutare se al controllo dei parametri biochimici corrisponde un effetto sugli outcomes clinici: riduzione della morbilità e mortalità cardiovascolare. Un altro studio (ADVANCE) multicentrico, randomizzato open label, che valuta gli effetti di Cinacalcet sulle calcificazioni vascolari dei pazienti in emodialisi, è in corso di pubblicazione. Alcuni dati preliminari sembrano confermare l'efficacia del calciomimetico + basse dosi di vitamina $\mathrm{D}$ nell'attenuare la progressione delle calcificazioni valvolari cardiache rispetto a un regime a base di dosi variabili di vitamina D (30). É, comunque, ancora assai presto per trarne conclusioni definitive.

Secondo il mio parere, il calciomimetico è indispensabile somministrarlo nei pazienti con IPT severo anche in monoterapia, se presente ipercalcemia e/o iperfosforemia. Altrimenti nei pazienti con IPT di medio grado deve essere associato ad analoghi della vitamina D (paracalcitolo). Come primo approccio a un iniziale e persistente incremento dei livelli di PTH ritengo opportuno somministrare analoghi della vitamina D (paracalcitolo). Il calciomimetico deve essere considerato pertanto un farmaco di grande efficacia, discretamente tollerato, che ha permesso, in modo sostanziale, di ridurre l'incidenza della paratiroidectomia.

\section{Bibliografia}

1. Liu S, Tang W, ZHO U, et al. Fibroblastic growth factor 23 is counter-regolatory phosphaturic hormone for vitamin D. J Am Soc Nephrol 2006; 17: 1305-15.

2. Silver S, Naveh-Many T. Phosphate and the parathyroid. Kidney Int 2009; 75: 898-905.

3. Nabeshima Y, Imura H. $\alpha$ Klotho: A regulator that integrates calcium homeostasis. Am J Nephrol 2008; 28: 455-64.

4. Krajisnik T, Bjorklund P, Marsell R, et al. Fibroblast growth factor-23 regulates parathyroid hormone and $1 \alpha$-hydroxylase expression in cultured bovine parathyroid cells. J Endocrinol 2007; 195: 125-31.

5. Ben-Dov IZ, Galitzer H, Lavi-Moshayoff V, et al. The parathyroid is a target organ for FGF23 in rats. J Clin Invest 2007; 117(12): 4003-8.

6. Gutierrez OM. Fibrobastic Growth factor 23 and disordered vitamin $\mathrm{D}$ metabolism in chronic kidney disease: Updating the "Trade Off" hypothesis". Clin J Am Soc Nephrol 2010; 5 (9): 1710-6.

7. Ix JH. Fibroblastic growth factor and early decrements of renal function: the heart and soul study. Nephrol Dial Transplant 2010; 25 (3): 993-7.

8. Evenepoel P, Meijers B, Viaene L, et al. Fibroblastic growth factor-23 in early chronic kidney disease; additional 
support in favour of a phosphate-centric paradigm for the pathogenesis of secondary hyperparathyroidism. Clin J Am Soc Nephrol 2010; 5 (7): 1268-76.

9. Oliveira RB, Cancela Al, Graciolli FG, et al. Early control of PTH and FGF23 in normophosphatemic CKD patients: A new target in CKD-MBD therapy? Clin J Am Soc Nephrol 2010; 5: 286-91.

10. Pereira RC, Juppner H, Azucena-Serrano CE, et al. Patterns of FGF-23, DMP1, and MEPE expression in patients with chronic kidney disease. Bone 2009; 45: 1161-68.

11. Komaba H, Goto S, Fujii H, et al. Depressed expression of Klotho and FGF receptor 1 in hyperplastic parathyroid glands for uremic patients. Kidney Int 2010; 77 (3): 232-8.

12. Kumata $\mathrm{C}$, Mizobuchi $\mathrm{M}$, Ogata $\mathrm{H}$, et al. Involvement of alpha-Klotho and fibroblast growth factor receptor in the development of secondary hyperparathyroidism. Am J Nephrol 2010; 31 (3): 230-8.

13. Canalejo R, Canalejo A, Martinez-Moreno JM, et al. FGF23 fails to inhibit parathyroid glands. J Am Soc Nephrol 2010; 21: 1125-35.

14. Galitzer H, Ben-Dov IZ, Silver J, et al. Parathyroid cell resistance to fibroblstic growth factor 23 in secondary hyperparathyroidism of chronic kidney disease. Kidney Int 2010; 77 (3): 211-8.

15. Isakova T, Gutierrez OM, Smith K, et al. Pilot study of dietary phosphorus restriction and phosphorus binders to target fibroblast growth factor 23 in patients with chronic kidney disease. Nephrol Dial Transplant 2010; Jul 14 (Epub ahead of print).

16. Gonzales EA, Sachdeva A, Oliver DA, et al. Vitamin D insufficiency and deficiency in chronic kidney disease. A single center observational study. Am J Nephrol 2004; 24: 503-10.

17. Kidney Disease: Improving Global Outcomes (KDIGO) CKD-MBD Work Goup. KDIGO clinical practice guideline for the diagnosis, evaluation, prevention and treatment of Chronic Kidney Disease-Mineral and Bone Disorder(CKD-MBD). Kidney Int 2009; 113 (Suppl), S1130.

18. Coen G, Mantella D, Manni M, et al. 25-hydroxyvitamin leves and bone histomorphometry in hemodialysis renal osteodystrophy. Kidney Int 2005; 68: 1840-8.

19. Autier P, Gandini S. Vitamin D supplementation and total mortality. Arch Intern Med 2007; 167: 1730-37.

20. Kooienga L, Fried L, Scragg R, et al. The effect of combined calcium and Vitamin D3 supplementation on serum intact parathyroid hormone in moderate CKD. Am J Kidney Dis 2009; 53 (3): 408-16.

21. Coyne D, Acharya M, Qiu P, et al. Paricalcitol capsule for the treatment of secondary hyperparathyroidism in stages 3 and 4. Am J Kidney Dis 2006; 47: 263-76.

22. Levin A, Bakris GL, Molitch, et al. Prevalence of abnormal serum vitamin D, PTH, calcium, and phosphorus in patients with chronic kidney disease: results of the study to evaluate early kidney disease. Kidney Int 2007; 71: 31-8.

23. Agarwal R, Acharya M, Tuian J, et al. Antiproteinuric effect of paracalcitol in chronic kidney disease. Kidney Int 2005; 68: 2823-38.

24. Slatopolsky E, Cozzolino M, Finch JL. Differential effect of 19-Nor-1,25-(OH)2D2 and 1alpha-hydroxyvitamin D2, on calcium and phosphorus in normal and uremic rats. Kidney Int 2002; 62(4): 1277-84.

25. Souberbille JLP, Roth H, Fougere DP, et al. Parathyroid hormone measurement in CKD. Kidney Int 2010; 77: 93100.

26. Marangella M. PTH a che punto siamo con il dosaggio? Giornale Italiano di Nefrologia, 2009; 5: 600 -7.

27. Messa P, Macario F, Yaqoob M, et al. The OPTIMA study: assessing a new Cinacalcet treatment algorithm for secondary hyperparathyroidism. Clin J Am Soc Nephrol 2008; 3: 36-45.

28. Fishbane S, Shapiro WB, Corry DB, et al. Cinacalcet HCL and concurrent low dose of vitamin D sterols in patients with moderate to severe secondary hyperparathyroidism in dialysis patients comparing with vitamin $\mathrm{D}$ alone. The ACHIEVE Study results. Clin J Am Soc Nephrol 2008; 3: 1718-25.

29. Urena P, Jacobson SH, Zitt E, et al. Cinacalcet and achievement of the NKF/K-DOQI recommended target values for bone and mineral metabolism in real world clinical opractice- The ECHO observational study. Nephrol Dial Transplant 2009; 24 (9): 2852-9.

30. Floege J, Raggi P, BlocK GA, et al. Study design and subject baseline characteristics in the ADVANCE Study: effects of Cinacalcet on vascular calcification in hemodialysis patients. Nephrol Dial Transplant 2010; 1-8. 